Xu Fan, Ke-Xiang Liu, Hong-Yu Zhu, Zhong-Yan Li* and Lin Yuan*

\title{
The crystal structure of $2,2^{\prime}-\left(\left(1 E, 1^{\prime} E\right)\right.$-hydrazine- 1,2-diylidenebis(methaneylylidene)) bis(4-chlorophenol), $\mathrm{C}_{14} \mathrm{H}_{10} \mathrm{Cl}_{2} \mathrm{~N}_{2} \mathrm{O}_{2}$
}

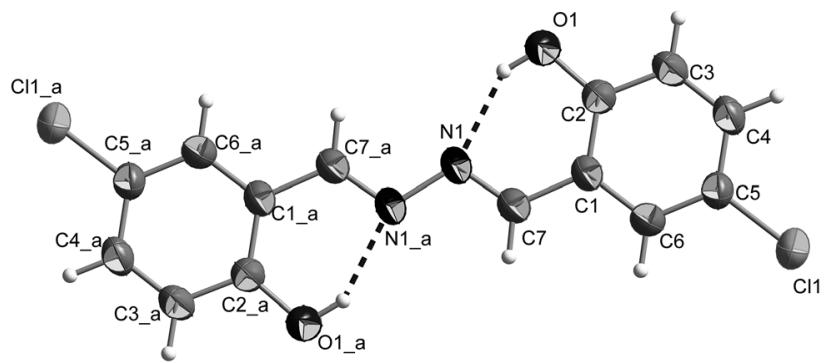

https://doi.org/10.1515/ncrs-2021-0438

Received November 15, 2021; accepted December 17, 2021;

published online January 5, 2022

\section{Abstract}

$\mathrm{C}_{14} \mathrm{H}_{10} \mathrm{Cl}_{2} \mathrm{~N}_{2} \mathrm{O}_{2}$, triclinic, $P \overline{1}$ (no. 2), $a=6.165(12) \AA$, $b=7.192(13) \AA, c=7.829(15) \AA, \alpha=74.97(2)^{\circ}, \beta=81.92(2)^{\circ}$, $y=79.84(2)^{\circ}, V=328.3(11) \AA^{3}, Z=1, R_{g t}(F)=0.0312$, $w R_{\text {ref }}\left(F^{2}\right)=0.0859, \mathrm{~T}=296(2) \mathrm{K}$.

\section{CCDC no.: 2107221}

The molecular structure is shown in the figure. Table 1 contains crystallographic data and Table 2 contains the list of the atoms including atomic coordinates and displacement parameters.

\section{Source of materials}

5-Chlorosalicylaldehyde (1.56 g, $10 \mathrm{mmol})$, hydrazine hydrate $(0.25 \mathrm{~g}, 5 \mathrm{mmol})$ and ethanol $(30 \mathrm{~mL})$ were heated and stirred at $353 \mathrm{~K}$ for $8 \mathrm{~h}$. Then the solvent was concentrated and filtered. The crude product was recrystallized from ethanol to afford yellow crystals ( $1.31 \mathrm{~g}$, yield $85 \%$ ).

*Corresponding authors: Zhong-Yan Li and Lin Yuan, College of Chemistry and Bioengineering, Hunan University of Science and Engineering, Yongzhou, Hunan 425199, P. R. China, E-mail: lizhongyandongdong@126.com (Z.-Y. Li) and tcyl431102@163.com (L. Yuan). https://orcid.org/0000-0002-69550161 (L. Yuan)

Xu Fan, Ke-Xiang Liu and Hong-Yu Zhu, College of Chemistry and Bioengineering, Hunan University of Science and Engineering, Yongzhou, Hunan 425199, P. R. China
Table 1: Data collection and handling.

\begin{tabular}{|c|c|}
\hline Crystal: & Yellow block \\
\hline Size: & $0.41 \times 0.38 \times 0.33 \mathrm{~mm}$ \\
\hline Wavelength: & Mo K $\alpha$ radiation $(0.71073 \AA)$ \\
\hline$\mu:$ & $0.50 \mathrm{~mm}^{-1}$ \\
\hline Diffractometer, scan mode: & Bruker APEX-II, $\varphi$ and $\omega$ \\
\hline$\theta_{\max }$, completeness: & $25.0^{\circ},>99 \%$ \\
\hline$N(h k l)_{\text {measured }}, N(h k l)_{\text {unique }}, R_{\text {int }}:$ & $3150,1160,0.013$ \\
\hline Criterion for $I_{\mathrm{obs}}, N\left(h k l_{\mathrm{gt}}\right.$ : & $I_{\text {obs }}>2 \sigma\left(I_{\text {obs }}\right), 1028$ \\
\hline$N(\text { param })_{\text {refined }}:$ & 92 \\
\hline Programs: & Bruker [1], SHELX [2, 3] \\
\hline
\end{tabular}

\section{Experimental details}

All hydrogen atoms were identified in difference Fourier syntheses. The $U_{\text {iso }}$ values of the hydrogen atoms of phenolic hydroxyl groups were set to $1.5 U_{e q}(\mathrm{C})$ and the $U_{\text {iso }}$ values of all other hydrogen atoms were set to $1.2 U_{e q}(\mathrm{C})$.

\section{Comment}

Schiff bases are a class of compounds containing the imino group $-\mathrm{C}=\mathrm{N}-$, which have important chemical and biological properties [4, 5]. Schiif bases may have

Table 2: Fractional atomic coordinates and isotropic or equivalent isotropic displacement parameters $\left(\AA^{2}\right)$.

\begin{tabular}{lrrrr}
\hline Atom & $\boldsymbol{x}$ & $\boldsymbol{y}$ & $\boldsymbol{z}$ & $\boldsymbol{U}_{\text {iso }}{ }^{*} / \boldsymbol{U}_{\text {eq }}$ \\
\hline C1 & $0.4596(3)$ & $0.3200(2)$ & $0.3872(2)$ & $0.0407(4)$ \\
C2 & $0.6783(3)$ & $0.2419(2)$ & $0.4198(2)$ & $0.0445(4)$ \\
C3 & $0.7262(3)$ & $0.1472(3)$ & $0.5905(2)$ & $0.0510(4)$ \\
H3 & 0.871152 & 0.092328 & 0.611733 & $0.061^{*}$ \\
C4 & $0.5626(3)$ & $0.1333(2)$ & $0.7286(2)$ & $0.0503(4)$ \\
H4 & 0.596520 & 0.070425 & 0.843404 & $0.060^{*}$ \\
C5 & $0.3478(3)$ & $0.2124(2)$ & $0.6973(2)$ & $0.0459(4)$ \\
C6 & $0.2954(3)$ & $0.3033(2)$ & $0.5293(2)$ & $0.0455(4)$ \\
H6 & 0.149081 & 0.354310 & 0.509709 & $0.055^{*}$ \\
C7 & $0.3993(3)$ & $0.4212(2)$ & $0.2121(2)$ & $0.0446(4)$ \\
H7 & 0.251233 & 0.468702 & 0.196384 & $0.053^{*}$ \\
Cl1 & $0.14214(8)$ & $0.19598(8)$ & $0.87486(6)$ & $0.0676(2)$ \\
N1 & $0.5439(2)$ & $0.4472(2)$ & $0.07842(17)$ & $0.0469(4)$ \\
O1 & $0.8469(2)$ & $0.2561(2)$ & $0.28996(16)$ & $0.0632(4)$ \\
H1 & 0.798238 & 0.308576 & 0.194068 & $0.095^{*}$ \\
\hline
\end{tabular}


antibacterial, anti-inflammatory, anti-tumor and antiviral biological activities [6, 7]. Salicylaldehyde Schiff bases are an important class of chemical analysis reagents and organic ligands, and they are widely used in fluorescent probes [8]. We have also reported some Schiff bases of salicylaldehyde $[9,10]$.

The asymmetric unit of the title structure contains one half of the title molecule (see the figure). It can be clearly seen in the structure that the intra molecular hydrogen bonds are formed between the hydrogen atom of the phenolic hydroxyl group and the nitrogen atom of the imine. The molecule is in the trans configuration at the $\mathrm{C}(7)-\mathrm{N}(1)$ double bond (1.273(3) $\AA$ ). The bond angle of $\mathrm{N}(1)-\mathrm{C}(7)-\mathrm{C}(1)$ is $121.57(19)^{\circ}$. The geometric parameters of the title structure are all in the expected ranges [11].

Author contributions: All the authors have accepted responsibility for the entire content of this submitted manuscript and approved submission.

Research funding: This work was financially supported by Natural Science Foundation of Hunan Province of China (2021JJ30291), the Scientific Research Fund of Hunan Provincial Education Department (21A0518, 21C0691), Undergraduate Training Program for Innovation and Entrepreneurship of Hunan Province of China ([2021]197-3585), Undergraduate Training Program for Innovation and Entrepreneurship of Hunan University of Science and Engineering (2021), Yongzhou Guiding Science and Technology Plan Project (2021), the construct program of applied characteristic discipline in Hunan University of Science and Engineering.

Conflict of interest statement: The authors declare no conflicts of interest regarding this article.

\section{References}

1. Bruker. APEX3, SAINT-Plus, XPREP; Bruker AXS Inc.: Madison, Wisconsin, USA, 2016.

2. Sheldrick G. M. SHELXTL - integrated space-group and crystalstructure determination. Acta Crystallogr. 2015, A71, 3-8.

3. Sheldrick G. M. Crystal structure refinement with SHELXL. Acta Crystallogr. 2015, C71, 3-8.

4. Kumari S., Chauhan G. New cellulose-lysine Schiff-base-based sensor- adsorbent for mercury ions. ACS Appl. Mater. Interfaces 2014, 6, 5908-5917.

5. Sidi B. M. V., Elemine B., Aliyenne A., Hadou A., Daich A., Othman M., Lawson A. M. Highly enantioselective semisynthesis of $(+) /(-)$-Gossypol Schiff base derivatives from ground plant material. J. Nat. Prod. 2019, 82, 1779-1790.

6. Kolodziej B., Morawiak M., Schilf W., Kamieński B. Structure investigations of Schiff bases derived from 3-amino- $1 \mathrm{H}$ 1,2,4-triazole. J. Mol. Struct. 2019, 1184, 207-218.

7. Bigyan R. J., Jubaraj B. B. Recent progress in Schiff bases in detections of fluoride ions. Dyes Pigments 2021, 194, 109575.

8. Singh G., Sindhu J., Manisha Kumar V., Sharma V., Sharma S. K., Mehta S. K., Mahnashi M. H., Umar A., Kataria R. Development of an off-on selective fluorescent sensor for the detection of $\mathrm{Fe}^{3+}$ ions based on Schiff base and its Hirshfeld surface and DFT studies. J. Mol. Liq. 2019, 296, 111814.

9. Li Z.-Y., Jia G.-K., Yuan L., Bai P.-F., He H., Zhou Q. Syntheses, crystal structures and biological activities of three new Schiff bases derived from substituted salicylaldehyde and tris base. Chin. J. Struct. Chem. 2017, 36, 1797-1802.

10. Yuan L., Li Y.-D., Fan X., Li Z.-Y. The crystal structure of (E)- 2-((tertbutylimino)methyl)-4-chlorophenol, $\mathrm{C}_{11} \mathrm{H}_{14} \mathrm{CINO}$. Z. Kristallogr. $\mathrm{N}$. Cryst. Struct. 2021, 236, 1317-1318.

11. Safin D. A., Robeyns K., Garcia Y. Solid-state thermo- and photochromism in $N, N^{\prime}$-bis(5-X-salicylidene)diamines $(\mathrm{X}=\mathrm{H}, \mathrm{Br})$. RSC Adv. 2012, 2, 11379-11388. 